\title{
Conditional Least Squares and Copulae in Claims Reserving for a Single Line of Business
}

\author{
Michal Pešta ${ }^{\mathrm{a}, *}$, Ostap Okhrin ${ }^{\mathrm{b}}$ \\ ${ }^{a}$ Charles University in Prague, Faculty of Mathematics and Physics, Department of \\ Probability and Mathematical Statistics, Sokolovská 83, CZ-18675 Prague, Czech Republic \\ ${ }^{b}$ Humboldt University of Berlin, School of Business and Economics, Ladislaus von \\ Bortkiewicz Chair of Statistics, C.A.S.E. - Center for Applied Statistics and Economics, \\ Spandauer Strasse 1, D-10178 Berlin, Germany
}

\begin{abstract}
One of the main goals in non-life insurance is to estimate the claims reserve distribution. A generalized time series model, that allows for modeling the conditional mean and variance of the claim amounts, is proposed for the claims development. On contrary to the classical stochastic reserving techniques, the number of model parameters does not depend on the number of development periods, which leads to a more precise forecasting.

Moreover, the time series innovations for the consecutive claims are not considered to be independent anymore. Conditional least squares are used for model parameter estimation and consistency of such estimate is proved. Copula approach is used for modeling the dependence structure, which improves the precision of the reserve distribution estimate as well.

Real data examples are provided as an illustration of the potential benefits of the presented approach.
\end{abstract}

Keywords: claims reserving, reserve distribution, dependency modeling, copula, conditional least squares

JEL classification: C13, C32, C33, C53, G22

Subject Category and Insurance Branch Category: IM10, IM11, IM20, IM40

MSC classification: 60G10, 60G25, 60J20, 62H10, 62H20, 62J02, 62P05

\footnotetext{
*Corresponding author, tel: (+420) 221913 400, fax: (+420) 222323316

Email addresses: michal.pesta@mff.cuni.cz (Michal Pešta), ostap.okhrin@wiwi.hu-berlin.de (Ostap Okhrin)
}

Preprint submitted to 


\section{Introduction}

Claims reserving is one of the most important issues in general insurance. A large number of various methods has been invented, see England and Verrall (2002) or Wüthrich and Merz (2008) for an overview.

Main aim of this paper is to deal with serious issues in contemporary reserving techniques, which are quite often set aside, but cause serious problems in the actuarial estimation and prediction. Such pitfalls are assumption of independent claims, independent stochastic errors (or residuals) in the corresponding claims reserving model, and considering large number of parameters often depending on the number of observations.

Majority of the classical approaches are based on the assumption that the claim amounts in different years are independent. However, this assumption can sometimes be unrealistic or at least questionable. It has been pointed out that methods, which enable modeling the dependencies, are needed, cf. Antonio and Beirlant (2007) or Hudecová and Pešta (2013). The mentioned papers suggest the generalized linear mixed models (GLMM) or generalized estimating equations (GEE) to handle the possible dependence among the incremental claims in successive development years. These approaches extend the classical GLM and are frequently used in panel (longitudinal) data analyses. In this paper, we present another possible attitude, namely the conditional mean-variance model with a copula.

On one hand, time series model by Buchwalder et al. (2006) nicely and simply allow to model conditional mean and variance of the claim amounts. On the other hand, that model possesses two disadvantages, which are common for a huge majority of the reserving methods: infinite number of parameters (i.e., depending on the number of observation) and independent errors. Generally, large number of parameters decreases the precision of estimation, because of not sufficient amount of data for estimation. Furthermore, the classical statistical inference is not valid anymore when the number of parameters depends on the number of observation. To overcome such difficulties, we consider a generalized time series model with a finite number of parameters not depending on the number of development periods and, additionally, the model errors belonging to the same accident period are not independent.

Moreover, all the currently used bootstrap methods in claims reserving require independent residuals in order to estimate the distribution of the reserve and, consequently, calculate some distributional quantities, e.g., VaR 
at $99.5 \%$. Assumption of independent residuals can be quite unrealistic in the claims reserving setup. Hence, an alternative and more suitable resampling method needs to be proposed in order to sensibly estimate the reserves distribution.

Copulae have already been utilized in the claims reserving to model dependences between different lines of business, e.g., Shi and Frees (2011). On the contrary, it has to be emphasized that in our approach, only one line of business is taken into account. Copulae are therefore used to model dependences within claims corresponding to that single line of business. For sure, our approach can be generalized for several lines of business in the way that a second level of dependence (for instance, modeled again by the copulae) is introduced between the claim amounts from different lines of business.

The structure of this paper is as follows: The claims reserving notation is summarized in Section 2. In Section 3, a generalized time series model for the conditional mean and variance of claim amounts is introduced. Section 4 elaborates copula approach for dependence modeling within the generalized time series model for claims triangles. Section 5 covers estimation techniques for the parameters of the generalized time series model and copula as well. Consistency of the estimates is derived. Section 6 concerns prediction of the actuarial claims reserves and, furthermore, estimation of their distribution. Finally, all the presented methods and approaches are applied on real data in Section 7 in order to show their performance and outstanding benefits.

\section{Claims Reserving Notation}

We introduce the classical claims reserving notation and terminology. Outstanding loss liabilities are structured in so-called claims development triangles, see Table 1. Let us denote $Y_{i, j}$ all the claim amounts up to development year $j \in\{1, \ldots, n\}$ with accident year $i \in\{1, \ldots, n\}$. Therefore, $Y_{i, j}$ stands for the cumulative claims in accident year $i$ after $j$ development periods. The current year is $n$, which corresponds to the most recent accident year and development period as well. Hence, $Y_{i, j}$ is a random variable of which we have an observation if $i+j<n+1$ (a run-off triangle). That is, our data history consists of right-angled isosceles triangle $\left\{Y_{i, j}\right\}$, where $i=1, \ldots, n$ and $j=1, \ldots, n+1-i$. The diagonal elements $Y_{i, j}$, where $i+j$ is constant, correspond to the claim amounts in accounting year $i+j$.

The aim is to estimate the ultimate claims amount $Y_{i, n}$ and the outstand-

ing claims reserve $R_{i}^{(n)}=Y_{i, n}-Y_{i, n+1-i}$ for all $i=2, \ldots, n$. Additional to 


\begin{tabular}{|c|c|c|c|c|c|}
\hline \multirow{2}{*}{$\begin{array}{c}\text { Accident } \\
\text { year } i\end{array}$} & \multicolumn{5}{|c|}{ Development year $j$} \\
\hline & 1 & 2 & .. & $n-1$ & $n$ \\
\hline 1 & $Y_{1,1}$ & $Y_{1,2}$ & $\cdots$ & $Y_{1, n-1}$ & $Y_{1, n}$ \\
\hline 2 & $Y_{2,1}$ & $Y_{2,2}$ & $\cdots$ & $Y_{2, n-1}$ & \\
\hline$\vdots$ & $\vdots$ & $\vdots$ & $Y_{i, n+1-i}$ & & \\
\hline$n-1$ & $Y_{n-1,1}$ & $Y_{n-1,2}$ & & & \\
\hline$n$ & $Y_{n, 1}$ & & & & \\
\hline
\end{tabular}

Table 1: Run-off triangle for cumulative claim amounts $Y_{i, j}$.

that, it is needed to estimate the whole distribution of the reserves in order to provide important distributional quantities for the Solvency II purposes, e.g., quantiles for the value at risk calculation.

\section{Conditional Mean and Variance Model}

Run-off triangles are comprised by observations which are ordered in time. It is therefore natural to suspect the observations to be dependent. On one hand, the most natural approach is to assume that the observations of a common accident year are dependent. On the other hand, observations of different accident years are supposed to be independent. This assumption is similar to those of the Mack's chain ladder model, cf. Mack (1993).

$\mathcal{F}_{i, j}$ denotes the information set generated by trapezoid $\left\{Y_{k, l}: l \leq j, k \leq\right.$ $i+1-j\}$, i.e., $\mathcal{F}_{i, j}=\sigma\left(Y_{k, l}: l \leq j, k \leq i+1-j\right)$ is a filtration corresponding to the smallest $\sigma$-algebra containing historical claims with at most $j$ development periods paid in accounting period $i$ or earlier. This notation allows for zero or even negative index in filtration despite the fact that the claims corresponding to zero or negative development of accident years are not observed.

Let us define a nonlinear generalized semiparametric regression type of model. It can be considered as a generalization of the model proposed by Buchwalder et al. (2006). The first level of generalization is in the mean and variance structure, which was inspired by Patton (2012). The second 
level of generalization regarding the dependence structure will be introduced in the next Section 4 ,

Definition 1 (CMV model). The Conditional Mean and Variance (CMV) model assumes

$$
Y_{i, j}=\mu\left(Y_{i, j-1}, \boldsymbol{\alpha}, j\right)+\sigma\left(Y_{i, j-1}, \boldsymbol{\beta}, j\right) \varepsilon_{i, j}(\boldsymbol{\alpha}, \boldsymbol{\beta}),
$$

where $\boldsymbol{\alpha}$ and $\boldsymbol{\beta}$ are unknown parameters, which dimensions do not depend on $n, \mu$ is a continuous function in $\boldsymbol{\alpha}$ and $\sigma$ is a positive and continuous function in $\boldsymbol{\beta}$. Disturbances $\left\{\varepsilon_{i, j}(\boldsymbol{\alpha}, \boldsymbol{\beta})\right\}_{j=1}^{n+1-i}$ are independent sample copies of a stationary first-order Markov process for all $i$. All $\varepsilon_{i, j}(\boldsymbol{\alpha}, \boldsymbol{\beta})$ have the common true invariant distribution $G_{\boldsymbol{\alpha}, \boldsymbol{\beta}}$ which is absolutely continuous with respect to Lebesgue measure on the real line. Suppose that

$$
\begin{aligned}
\mathrm{E}\left[\varepsilon_{i, j}(\boldsymbol{\alpha}, \boldsymbol{\beta}) \mid \mathcal{F}_{i, j-1}\right] & =0, \\
\operatorname{Var}\left[\varepsilon_{i, j}(\boldsymbol{\alpha}, \boldsymbol{\beta}) \mid \mathcal{F}_{i, j-1}\right] & =s(\boldsymbol{\alpha}, \boldsymbol{\beta}),
\end{aligned}
$$

for all $i$ and $j$. Moreover for the unknown true values $\left[\boldsymbol{\alpha}^{* \top}, \boldsymbol{\beta}^{* \top}\right]^{\top}$ of parameters $\left[\boldsymbol{\alpha}^{\top}, \boldsymbol{\beta}^{\top}\right]^{\top}$, the conditional variance of errors equals one due to identifiability purposes, i.e., $s\left(\boldsymbol{\alpha}^{*}, \boldsymbol{\beta}^{*}\right)=1$.

The name of the model comes from the fact that the conditional mean and variance can be expressed as

$$
\begin{aligned}
\mathrm{E}\left[Y_{i, j} \mid \mathcal{F}_{i, j-1}\right] & =\mu\left(Y_{i, j-1}, \boldsymbol{\alpha}, j\right), \\
\operatorname{Var}\left[Y_{i, j} \mid \mathcal{F}_{i, j-1}\right] & =\sigma^{2}\left(Y_{i, j-1}, \boldsymbol{\beta}, j\right) s(\boldsymbol{\alpha}, \boldsymbol{\beta}) .
\end{aligned}
$$

This property allows for a wide variety of models for the conditional mean: types of ARMA models, vector autoregressions, linear and nonlinear regressions, and others. It also allows for a variety of models for the conditional variance: ARCH and any of its numerous parametric extensions (GARCH, EGARCH, GJR-GARCH, etc., see Bollerslev (2010)), stochastic volatility models, and others.

Patton (2012) considered a similar model, but the dependence was assumed in a different way. I.e., dependent copies of the time series (dependence between rows) were supposed, not dependent errors within each time series as we propose. Here, independent rows of errors $\left[\varepsilon_{i, 1}(\boldsymbol{\alpha}, \boldsymbol{\beta}), \ldots, \varepsilon_{i, n+1-i}(\boldsymbol{\alpha}, \boldsymbol{\beta})\right]$ imply independent rows of claims $\left[Y_{i, 1}, \ldots, Y_{i, n+1-i}\right]$. Moreover, the uncondi- 
tional mean and variance of the CMV model's errors equal the conditional ones: $\mathrm{E} \varepsilon_{i, j}(\boldsymbol{\alpha}, \boldsymbol{\beta})=0$ and $\operatorname{Var} \varepsilon_{i, j}(\boldsymbol{\alpha}, \boldsymbol{\beta})=s(\boldsymbol{\alpha}, \boldsymbol{\beta})$.

To provide an insight into possible candidates for the mean function $\mu$ and variance function $\sigma$, one may propose $\mu\left(Y_{i, j-1}, \boldsymbol{\alpha}, j\right)=\eta(\boldsymbol{\alpha}, j) Y_{i, j-1}$ and $\sigma\left(Y_{i, j-1}, \boldsymbol{\beta}, j\right)=\nu(\boldsymbol{\beta}, j) \sqrt{Y_{i, j-1}}$ or $\sigma\left(Y_{i, j-1}, \boldsymbol{\beta}, j\right)=\nu(\boldsymbol{\beta}, j) Y_{i, j-1}$. Sherman (1984) investigated decays $\eta(\boldsymbol{\alpha}, j)$, which should correspond to the link ratios. Hence, $\eta(\boldsymbol{\alpha}, j)$ should be decreasing in $j$ with limit 1 as $j$ tends to infinity: $1+\alpha_{1} \exp \left\{-\alpha_{2} j\right\}, 1+\alpha_{1} \exp \left\{-j^{\alpha_{2}}\right\}, 1+\alpha_{1} j^{-\alpha_{2}}, 1+\alpha_{1}\left(\alpha_{2}+j\right)^{-\alpha_{3}}, 1+\alpha_{1}^{-\alpha_{2}^{j}}$, $\alpha_{1}^{\alpha_{2}^{-j}},\left(1-\exp \left\{-\alpha_{1} j^{\alpha_{2}}\right\}\right)^{-1}, 1+\alpha_{1} \alpha_{2} j^{-1-\alpha_{2}} \exp \left\{\alpha_{1} j^{-\alpha_{2}}\right\}, \exp \left\{\alpha_{1} j^{-\alpha_{2}}\right\}, 1+$ $\alpha_{1} /\left(j+\alpha_{2}\right), 1+\alpha_{1} / \log \left(j+\alpha_{2}\right)$, etc., where $\alpha_{1}, \alpha_{2}, \alpha_{3}>0$. On the other hand, decay $\nu(\boldsymbol{\beta}, j)$ should be decreasing in $j$ with limit $0: \beta_{1} \exp \left\{-\beta_{2} j\right\}$, $\beta_{1} j^{-\beta_{2}}, \beta_{1} \log j \exp \left\{-\beta_{2} j\right\}, \beta_{1} j^{-\beta_{2}} \exp \left\{-\beta_{3} j\right\}, \beta_{1} \exp \left\{-\beta_{2} j^{2}\right\}, \beta_{1} /\left(j+\beta_{2}\right)$, $\beta_{1} / \log \left(j+\beta_{2}\right), \beta_{1} j^{-\beta_{2}} \exp \left\{-\beta_{3} j^{2}\right\}$, etc., where $\beta_{1}, \beta_{2}, \beta_{3}>0$.

In actuarial praxis, these decays are quite often used, mainly for projecting the development (forecasting of the claim amounts after $n$ development periods). Despite of that, the parameters of the decay curves are not estimated directly from the triangle, but the chain ladder estimates $\widehat{f}_{j}$ and $\widehat{\sigma}_{j}^{2}$ of the development factors $f_{j}$ and the nuisance variance parameters $\sigma_{j}^{2}$ (Mack, 1993) are smoothed and used for the decay parameters estimation. This two-step procedure does not assure that the estimated decay parameters will be at least asymptotically unbiased. Unlike that, we will estimate the parameters directly from the data triangle and prove the estimates' consistency.

Sherman (1984) assumed independence of individual link ratios (development factors) when estimating the decay parameters by parametric curve fitting. We relax the independence assumption and model the link ratios conditionally having dependent errors.

When comparing the CMV model with the model investigated by Buchwalder et al. (2006), two main differences arise. The CMV model allows for dependent errors and assumes finite number of parameters not depending on number of development periods $n$. Indeed, the CMV model requires known functions with unknown finite dimensional parameters. Parameters of the time series model from Buchwalder et al. (2006) are $\left\{f_{j}\right\}_{j=1}^{n}$ and $\left\{\sigma_{j}\right\}_{j=1}^{n}$, which play the role of $\eta(\boldsymbol{\alpha}, j)$ and $\nu(\boldsymbol{\beta}, j)$, respectively. It is important to note that the classical stochastic inference is not valid in the setup, when number of parameters depends on the number of observation. Thus, legitimacy of the bootstrap procedure in that case is questionable.

Furthermore in the chain ladder, the estimate for $f_{n-1}$ is just a pure ratio 
of two random variables and, moreover to estimate $\sigma_{n-1}^{2}$, only doubtful adhoc estimates were proposed due to the fact that the claims triangle simply does not contain data for a reasonable estimate (e.g., a consistent one).

\section{Dependence Modeling by Copulae}

Since the mean and variance trend are removed by the CMV model, the rest of the relationship among claim amounts can be additionally captured by modeling dependent errors. The inspiration for the dependence structure was taken from Chen and Fan (2006).

Assumption $C .\left\{\varepsilon_{i, j}(\boldsymbol{\alpha}, \boldsymbol{\beta})\right\}_{j=1}^{n+1-i}$ are independent sample copies of a stationary first-order Markov process for all $i$ generated from $\left(G_{\boldsymbol{\alpha}, \boldsymbol{\beta}}(\cdot), C(\cdot, \cdot ; \boldsymbol{\gamma})\right)$, where $C(\cdot, \cdot ; \boldsymbol{\gamma})$ is the true parametric copula for $\left[\varepsilon_{i, j-1}(\boldsymbol{\alpha}, \boldsymbol{\beta}), \varepsilon_{i, j}(\boldsymbol{\alpha}, \boldsymbol{\beta})\right]$, which is given and fixed up to unknown parameter $\gamma$ and is absolutely continuous with respect to Lebesgue measure on $[0,1]^{2}$.

It is believed that there exist a kind of information overlap between the claims from consecutive development periods, which corresponds to the dependence between the CMV model's errors modeled by copulae.

Assumption $\mathrm{C}$ together with the CMV model yield a copula-based model, where the joint bivariate distribution of errors $\left[\varepsilon_{i, j-1}(\boldsymbol{\alpha}, \boldsymbol{\beta}), \varepsilon_{i, j}(\boldsymbol{\alpha}, \boldsymbol{\beta})\right]$ has the following distribution function

$$
H\left(e_{1}, e_{2}\right)=C\left(G_{\boldsymbol{\alpha}, \boldsymbol{\beta}}\left(e_{1}\right), G_{\boldsymbol{\alpha}, \boldsymbol{\beta}}\left(e_{2}\right) ; \boldsymbol{\gamma}\right)
$$

Then, the conditional copula density can be derived as

$$
h\left(e_{2} \mid e_{1}\right)=g_{\boldsymbol{\alpha}, \boldsymbol{\beta}}\left(e_{2}\right) c\left(G_{\boldsymbol{\alpha}, \boldsymbol{\beta}}\left(e_{1}\right), G_{\boldsymbol{\alpha}, \boldsymbol{\beta}}\left(e_{2}\right) ; \boldsymbol{\gamma}\right),
$$

where $c$ is the copula density a $g_{\boldsymbol{\alpha}, \boldsymbol{\beta}}$ is the marginal density corresponding to the univariate distribution function $G_{\boldsymbol{\alpha}, \boldsymbol{\beta}}$. The latter relation (3) will play an important role in "making" the dependent errors conditionally independent during the forthcoming estimation and prediction process.

\section{Parameter Estimation}

The CMV model from Definition 1 together with the copula Assumption $\mathrm{C}$ contain three vector parameters, which need to be estimated. The estimation process consists of two stages. In the first one, mean and variance 
parameters $\boldsymbol{\alpha}$ and $\boldsymbol{\beta}$ are estimated in a distribution-free fashion, since no specific distributional assumptions are proposed nor required for the claims. The second stage concerns estimation of the dependence structure, mainly the copula parameter $\gamma$, in a likelihood based way.

\subsection{Estimation in CMV Model}

Since the CMV model is defined in a conditional style, conditional least squares (CLS) of the sample centered conditional moments of the claims are minimized in order to obtain estimates of the CMV model parameters.

Definition 2 (Conditional least squares estimates). Let us denote

$$
M_{n}(\boldsymbol{\alpha}, \boldsymbol{\beta})=\frac{1}{n-1} \sum_{j=2}^{n} \frac{1}{n+1-j} \sum_{i=1}^{n+1-j} \frac{\left[Y_{i, j}-\mu\left(Y_{i, j-1}, \boldsymbol{\alpha}, j\right)\right]^{2}}{\sigma^{2}\left(Y_{i, j-1}, \boldsymbol{\beta}, j\right)}
$$

and

$$
\begin{aligned}
& V_{n}(\boldsymbol{\alpha}, \boldsymbol{\beta}) \\
& =\frac{1}{n-1} \sum_{j=2}^{n} \frac{1}{n+1-j} \sum_{i=1}^{n+1-j}\left\{\left[Y_{i, j}-\mu\left(Y_{i, j-1}, \boldsymbol{\alpha}, j\right)\right]^{2}-\sigma^{2}\left(Y_{i, j-1}, \boldsymbol{\beta}, j\right)\right\}^{2},
\end{aligned}
$$

where parameters $\boldsymbol{\alpha}$ and $\boldsymbol{\beta}$ belong to parameter spaces $\boldsymbol{\Theta}_{1}$ and $\boldsymbol{\Theta}_{2}$. The conditional least squares estimate of the mean parameter $\boldsymbol{\alpha}$ for a fixed value of parameter $\boldsymbol{\beta} \in \boldsymbol{\Theta}_{2}$ is defined as

$$
\widehat{\boldsymbol{\alpha}}(\boldsymbol{\beta})=\arg \min _{\boldsymbol{\alpha} \in \boldsymbol{\Theta}_{1}} M_{n}(\boldsymbol{\alpha}, \boldsymbol{\beta})
$$

and the conditional least squares estimate of the variance parameter $\boldsymbol{\beta}$ for a fixed value of parameter $\boldsymbol{\alpha} \in \boldsymbol{\Theta}_{1}$ is defined as

$$
\widehat{\boldsymbol{\beta}}(\boldsymbol{\alpha})=\arg \min _{\boldsymbol{\beta} \in \boldsymbol{\Theta}_{2}} V_{n}(\boldsymbol{\alpha}, \boldsymbol{\beta}) .
$$

The reason, why the parameter estimates for the CMV model are defined as above, lies in the fact that it is computationally not feasible to find the global minimum of $M_{n}$ and $V_{n}$ with respect to $\left[\boldsymbol{\alpha}^{\top}, \boldsymbol{\beta}^{\top}\right]^{\top}$ simultaneously.

The forthcoming theory (Theorems 1, 2, and Corollary 3) assures that the CLS estimates are reasonable and, moreover, consequent Algorithm 1 provides a computational way for obtaining CMV parameter estimates. 
Theorem 1 (Conditional least squares consistency for the mean). Let Model $C M V$ hold and $\boldsymbol{\beta} \in \mathbf{\Theta}_{2}$ be fixed. Assume that

(i) $\left\{\left[Y_{i, j}-\mu\left(Y_{i, j-1}, \boldsymbol{\alpha}, j\right)\right]^{2} / \sigma^{2}\left(Y_{i, j-1}, \boldsymbol{\beta}, j\right)\right\}_{i, j \in \mathbb{N}}$ is uniformly integrable,

(ii) for all $\boldsymbol{\alpha}, \boldsymbol{\alpha}^{\prime} \in \boldsymbol{\Theta}_{1}$ and $n \in \mathbb{N}$,

$$
\left|\left[Y_{i, j}-\mu\left(Y_{i, j-1}, \boldsymbol{\alpha}, j\right)\right]^{2}-\left[Y_{i, j}-\mu\left(Y_{i, j-1}, \boldsymbol{\alpha}^{\prime}, j\right)\right]^{2}\right| \stackrel{a . s .}{\leq} C_{j} g\left(\left\|\boldsymbol{\alpha}-\boldsymbol{\alpha}^{\prime}\right\|\right),
$$

where $\left\{C_{j}\right\}_{j \in \mathbb{N}}$ is a stochastic sequence not depending on $\boldsymbol{\alpha}$ such that $C_{j}=\mathcal{O}_{\mathrm{P}}\left(\sigma^{2}\left(Y_{i, j-1}, \boldsymbol{\beta}, j\right)\right), j \rightarrow \infty$ for all $i \in \mathbb{N}$ and $g$ is nonstochastic such that $g(t) \downarrow 0$ as $t \downarrow 0$,

(iii) $s(\cdot, \boldsymbol{\beta})$ is a Lipschitz function on the compact parameter space $\boldsymbol{\Theta}_{1}$ such that the true unknown parameter $\boldsymbol{\alpha}^{*}(\boldsymbol{\beta})$ is its unique global minimum.

Then $\widehat{\boldsymbol{\alpha}}(\boldsymbol{\beta}) \underset{n \rightarrow \infty}{\stackrel{\mathrm{P}}{\longrightarrow}} \boldsymbol{\alpha}^{*}(\boldsymbol{\beta})$.

Proof. Let us define

$$
A_{n, j}:=\frac{1}{(n-1)(n+1-j)} \sum_{i=1}^{n+1-j} \frac{\left[Y_{i, j}-\mu\left(Y_{i, j-1}, \boldsymbol{\alpha}, j\right)\right]^{2}}{\sigma^{2}\left(Y_{i, j-1}, \boldsymbol{\beta}, j\right)}
$$

Since $\mathrm{E}\left[A_{n, j} \mid \mathcal{F}_{n, j-1}\right]=\frac{1}{n-1} s(\boldsymbol{\alpha}, \boldsymbol{\beta})$, then $A_{n, j}-\mathrm{E}\left[A_{n, j} \mid \mathcal{F}_{n, j-1}\right]=A_{n, j}-\mathrm{E} A_{n, j}$ is a martingale difference array with respect to filtration $\mathcal{F}_{n, j}$. Moreover, if $\left[Y_{i, j}-\mu\left(Y_{i, j-1}, \boldsymbol{\alpha}, j\right)\right]^{2} / \sigma^{2}\left(Y_{i, j-1}, \boldsymbol{\beta}, j\right)$ is uniformly integrable, then $(n-$ 1) $A_{n, j}$ is uniformly integrable as well. This allows to apply the $\mathrm{L}_{1}$ law of large numbers for the martingale difference arrays (Davidson, 1994, Theorem 19.7). Hence, $\sum_{j=2}^{n}\left[A_{n, j}-\frac{1}{n-1} s(\boldsymbol{\alpha}, \boldsymbol{\beta})\right] \underset{n \rightarrow \infty}{\stackrel{\mathrm{L}_{1}}{\longrightarrow}} 0$, which implies $\sum_{j=2}^{n} A_{n, j} \underset{n \rightarrow \infty}{\stackrel{\mathrm{P}}{\longrightarrow}}$ $s(\boldsymbol{\alpha}, \boldsymbol{\beta})$. According to the definition of $A_{n, j}$, we have obtained the weak law of large numbers (WLLN) for $M_{n}(\boldsymbol{\alpha}, \boldsymbol{\beta})$ for all $\boldsymbol{\alpha} \in \boldsymbol{\Theta}_{1}$ and $\boldsymbol{\beta} \in \boldsymbol{\Theta}_{2}$ (pointwise).

Assumption (4) gives a Lipschitz-type condition for $M_{n}(\cdot, \boldsymbol{\beta})$, i.e.,

$$
\left|M_{n}(\boldsymbol{\alpha}, \boldsymbol{\beta})-M_{n}\left(\boldsymbol{\alpha}^{\prime}, \boldsymbol{\beta}\right)\right| \leq K_{n} g\left(\left\|\boldsymbol{\alpha}-\boldsymbol{\alpha}^{\prime}\right\|\right), \quad \text { a.s. }
$$

for all $\boldsymbol{\alpha}, \boldsymbol{\alpha}^{\prime}$ and $K_{n}=\mathcal{O}_{\mathrm{P}}(1), n \rightarrow \infty$. Combining this fact with $s(\cdot, \boldsymbol{\beta})$ being Lipschitz, then $\left\{M_{n}(\cdot, \boldsymbol{\beta})-s(\cdot, \boldsymbol{\beta})\right\}_{n \in \mathbb{N}}$ is stochastically equicontinuous 
for all $\boldsymbol{\beta}$ by Davidson (1994, Theorem 21.10). Furthermore, Theorem 21.9 by Davidson (1994) provides the weak uniform law of large numbers (WULLN) for $M_{n}(\boldsymbol{\alpha}, \boldsymbol{\beta})$ in $\boldsymbol{\alpha}$ for all $\boldsymbol{\beta} \in \boldsymbol{\Theta}_{2}$, i.e.,

$$
\sup _{\boldsymbol{\alpha} \in \boldsymbol{\Theta}_{1}}\left|M_{n}(\boldsymbol{\alpha}, \boldsymbol{\beta})-s(\boldsymbol{\alpha}, \boldsymbol{\beta})\right| \underset{n \rightarrow \infty}{\stackrel{\mathrm{P}}{\longrightarrow}} 0
$$

for all $\boldsymbol{\beta} \in \boldsymbol{\Theta}_{2}$.

Taking into account that continuous (or, moreover, Lipschitz) functions reach their global extremes on a compact set, Theorem 4.2.1 by Bierens (1994) yields the desired weak consistency of the $\boldsymbol{\alpha}$ parameter.

Weak consistency (in probability) of mean parameter estimate is shown, but also the strong version (almost sure convergence) can be provided. It would require $C_{j}$ to be bounded almost surely, which is less feasible.

Lipschitz kind of assumption (ii) can be replaced by a stronger one: uniform equiboundedness in probability. In that case, it suffices to assume $\mathrm{E}_{\sup _{\boldsymbol{\alpha} \in \boldsymbol{\Theta}_{1}}}\left\|\nabla_{\boldsymbol{\alpha}} M_{n}(\boldsymbol{\alpha}, \boldsymbol{\beta})\right\|<\Delta_{1}$ for all $n$ and $\boldsymbol{\beta}$ and convexity of the compact parameter space $\Theta_{1}$ for applying the stochastic mean-value theorem. The compactness of the parameter space can even be relaxed to its total boundedness.

Similar theorem as above is going to be postulated for the CLS variance parameter estimate to ensure its appropriateness. Firstly, let us define

$$
\begin{aligned}
& B_{n, j}(\boldsymbol{\alpha}, \boldsymbol{\beta}) \\
& :=\frac{1}{(n-1)(n+1-j)} \sum_{i=1}^{n+1-j}\left\{\left[Y_{i, j}-\mu\left(Y_{i, j-1}, \boldsymbol{\alpha}, j\right)\right]^{2}-\sigma^{2}\left(Y_{i, j-1}, \boldsymbol{\beta}, j\right)\right\}^{2}
\end{aligned}
$$

and

$$
v(\boldsymbol{\alpha}, \boldsymbol{\beta}):=\lim _{n \rightarrow \infty} \sum_{j=2}^{n} \mathrm{E} B_{n, j}(\boldsymbol{\alpha}, \boldsymbol{\beta}) .
$$

Theorem 2 (Conditional least squares consistency for the variance). Let Model CMV hold and $\boldsymbol{\alpha} \in \boldsymbol{\Theta}_{1}$ be fixed. Assume that 
(i) random array

$$
\begin{aligned}
& \left\{\left\{\left[Y_{i, j}-\mu\left(Y_{i, j-1}, \boldsymbol{\alpha}, j\right)\right]^{2}-\sigma^{2}\left(Y_{i, j-1}, \boldsymbol{\beta}, j\right)\right\}^{2}\right. \\
& \left.\quad-\mathrm{E}\left\{\left[Y_{i, j}-\mu\left(Y_{i, j-1}, \boldsymbol{\alpha}, j\right)\right]^{2}-\sigma^{2}\left(Y_{i, j-1}, \boldsymbol{\beta}, j\right)\right\}^{2}\right\}_{i, j \in \mathbb{N}}
\end{aligned}
$$

is uniformly integrable,

(ii) for all $\boldsymbol{\beta}, \boldsymbol{\beta}^{\prime} \in \boldsymbol{\Theta}_{2}$ and $n \in \mathbb{N}$,

$$
\begin{aligned}
& \mid\left\{\left[Y_{i, j}-\mu\left(Y_{i, j-1}, \boldsymbol{\alpha}, j\right)\right]^{2}-\sigma^{2}\left(Y_{i, j-1}, \boldsymbol{\beta}, j\right)\right\}^{2} \\
& \quad-\left\{\left[Y_{i, j}-\mu\left(Y_{i, j-1}, \boldsymbol{\alpha}, j\right)\right]^{2}-\sigma^{2}\left(Y_{i, j-1}, \boldsymbol{\beta}^{\prime}, j\right)\right\}^{2} \mid \stackrel{\text { a.s. }}{\leq} D_{j} h\left(\left\|\boldsymbol{\beta}-\boldsymbol{\beta}^{\prime}\right\|\right),
\end{aligned}
$$

where $\left\{D_{j}\right\}_{j \in \mathbb{N}}$ is a stochastic sequence not depending on $\boldsymbol{\beta}$ such that $D_{j}=\mathcal{O}_{\mathrm{P}}(1), j \rightarrow \infty$ for all $i \in \mathbb{N}$ and $h$ is nonstochastic such that $h(t) \downarrow 0$ as $t \downarrow 0$,

(iii) for all $j \leq n, n \in \mathbb{N}$, and $m \in \mathbb{N}_{0}$,

$$
\mathrm{E}\left|\mathrm{E}\left[B_{n, j}(\boldsymbol{\alpha}, \boldsymbol{\beta})-\mathrm{E} B_{n, j}(\boldsymbol{\alpha}, \boldsymbol{\beta}) \mid \mathcal{F}_{n, j-m}\right]\right| \leq c_{n, j} d_{m}
$$

where $\left\{c_{n, j}\right\}_{j \leq n, n \in \mathbb{N}}$ and $\left\{d_{m}\right\}_{m \in \mathbb{N}_{0}}$ are constants such that $d_{m} \downarrow 0$ as $m \rightarrow \infty$,

(iv) $v(\boldsymbol{\alpha}, \cdot)$ is a Lipschitz function on the compact parameter space $\boldsymbol{\Theta}_{2}$ such that the true unknown parameter $\boldsymbol{\beta}^{*}(\boldsymbol{\alpha})$ is its unique global minimum.

Then $\widehat{\boldsymbol{\beta}}(\boldsymbol{\alpha}) \underset{n \rightarrow \infty}{\stackrel{\mathrm{P}}{\longrightarrow}} \boldsymbol{\beta}^{*}(\boldsymbol{\alpha})$.

Proof. The idea of this proof is similar as in the previous one. Note that $B_{n, j}(\boldsymbol{\alpha}, \boldsymbol{\beta})-\mathrm{E} B_{n, j}(\boldsymbol{\alpha}, \boldsymbol{\beta})$ is an $\mathrm{L}_{1}$-mixingale array with respect to filtration $\mathcal{F}_{n, j}$ due to (6). Condition (i) implies that $(n-1)\left[B_{n, j}(\boldsymbol{\alpha}, \boldsymbol{\beta})-\mathrm{E} B_{n, j}(\boldsymbol{\alpha}, \boldsymbol{\beta})\right]$ is uniformly integrable. This allows us to apply the $\mathrm{L}_{1}$ law of large numbers for the $\mathrm{L}_{1}$-mixingale arrays (Davidson, 1994, Theorem 19.11). Hence, $\sum_{j=2}^{n}\left[B_{n, j}(\boldsymbol{\alpha}, \boldsymbol{\beta})-\mathrm{E} B_{n, j}(\boldsymbol{\alpha}, \boldsymbol{\beta})\right] \underset{n \rightarrow \infty}{\stackrel{\mathrm{L}_{1}}{\longrightarrow}} 0$, which implies $\sum_{j=2}^{n} B_{n, j}(\boldsymbol{\alpha}, \boldsymbol{\beta}) \underset{n \rightarrow \infty}{\stackrel{\mathrm{P}}{\longrightarrow}}$ $v(\boldsymbol{\alpha}, \boldsymbol{\beta})$. According to the definition of $B_{n, j}(\boldsymbol{\alpha}, \boldsymbol{\beta})$, we have obtained the pointwise WLLN for $V_{n}(\boldsymbol{\alpha}, \boldsymbol{\beta})$ for all $\boldsymbol{\alpha} \in \boldsymbol{\Theta}_{1}$ and $\boldsymbol{\beta} \in \boldsymbol{\Theta}_{2}$. 
Assumption (5) gives a Lipschitz-type condition for $V_{n}(\boldsymbol{\alpha}, \cdot)$, i.e.,

$$
\left|V_{n}(\boldsymbol{\alpha}, \boldsymbol{\beta})-V_{n}\left(\boldsymbol{\alpha}, \boldsymbol{\beta}^{\prime}\right)\right| \leq D_{n}^{\prime} h\left(\left\|\boldsymbol{\beta}-\boldsymbol{\beta}^{\prime}\right\|\right), \quad \text { a.s. }
$$

for all $\boldsymbol{\beta}, \boldsymbol{\beta}^{\prime}$ and $D_{n}^{\prime}=\mathcal{O}_{\mathrm{P}}(1), n \rightarrow \infty$. Combining this fact with $v(\boldsymbol{\alpha}, \cdot)$ being Lipschitz, then $\left\{V_{n}(\boldsymbol{\alpha}, \cdot)-v(\boldsymbol{\alpha}, \cdot)\right\}_{n \in \mathbb{N}}$ is stochastically equicontinuous for all $\boldsymbol{\alpha}$ by Davidson (1994, Theorem 21.10). Furthermore, Theorem 21.9 by Davidson (1994) provides the WULLN for $V_{n}(\boldsymbol{\alpha}, \boldsymbol{\beta})$ in $\boldsymbol{\beta}$ for all $\boldsymbol{\alpha} \in \boldsymbol{\Theta}_{1}$, i.e.,

$$
\sup _{\boldsymbol{\beta} \in \boldsymbol{\Theta}_{2}}\left|V_{n}(\boldsymbol{\alpha}, \boldsymbol{\beta})-v(\boldsymbol{\alpha}, \boldsymbol{\beta})\right| \underset{n \rightarrow \infty}{\stackrel{\mathrm{P}}{\longrightarrow}} 0
$$

for all $\boldsymbol{\alpha} \in \boldsymbol{\Theta}_{1}$. Theorem 4.2.1 by Bierens (1994) yields the desired weak consistency of the $\boldsymbol{\beta}$ parameter.

A natural question arises: What is the connection between the true unknown parameter values $\boldsymbol{\alpha}^{*}$ and $\boldsymbol{\beta}^{*}$ of the CMV model and true unknown parameter values $\boldsymbol{\alpha}^{*}(\boldsymbol{\beta})$ and $\boldsymbol{\beta}^{*}(\boldsymbol{\alpha})$ from Theorems 1 and 22. The intuition behind the CMV model is that function $\mu$ should mimic the conditional mean of the claims and function $\sigma^{2}$ should model their conditional variance. Mathematically speaking, $\operatorname{Var}\left[Y_{i, j} / \sigma\left(Y_{i, j-1}, \boldsymbol{\beta}, j\right) \mid \mathcal{F}_{i, j-1}\right]$ and, similarly, $\operatorname{Var}\left[\left(Y_{i, j}-\mu\left(Y_{i, j-1}, \boldsymbol{\alpha}, j\right)\right)^{2} \mid \mathcal{F}_{i, j-1}\right]$ should be as small as possible. Taking into account that the data triangle does not possess the same number of claim amounts entries for each development period $j$, it is reasonable to assume that if the CMV model holds, then both discrepancy measures

$$
\lim _{n \rightarrow \infty} \mathrm{E}\left\{\frac{1}{n-1} \sum_{j=2}^{n} \frac{1}{n+1-j} \sum_{i=1}^{n+1-j} \operatorname{Var}\left[\frac{Y_{i, j}}{\sigma\left(Y_{i, j-1}, \boldsymbol{\beta}, j\right)} \mid \mathcal{F}_{i, j-1}\right]\right\}
$$

and

$$
\lim _{n \rightarrow \infty} \mathrm{E}\left\{\frac{1}{n-1} \sum_{j=2}^{n} \frac{1}{n+1-j} \sum_{i=1}^{n+1-j} \operatorname{Var}\left[\left(Y_{i, j}-\mu\left(Y_{i, j-1}, \boldsymbol{\alpha}, j\right)\right)^{2} \mid \mathcal{F}_{i, j-1}\right]\right\}
$$

reach their global minimum just at the same true unknown parameter values $\boldsymbol{\alpha}^{*}$ and $\boldsymbol{\beta}^{*}$ of the CMV model. However, measures (7) and (8) are nothing else than $s(\boldsymbol{\alpha}, \boldsymbol{\beta})$ and $v(\boldsymbol{\alpha}, \boldsymbol{\beta})$. Now, let us define the interior of set $\boldsymbol{\Theta}$ by $\stackrel{\circ}{\Theta}$. 
Corollary 3 (Consistency of the CLS estimates). Suppose that the assumptions of Theorems 1 and 2 hold. Let $s \in \mathcal{C}^{2}\left(\boldsymbol{\Theta}_{1} \times \boldsymbol{\Theta}_{2}\right), v \in \mathcal{C}^{2}\left(\boldsymbol{\Theta}_{1} \times \boldsymbol{\Theta}_{2}\right)$, and both functions $s$ and $v$ have their unique global minimum on compact set $\boldsymbol{\Theta}_{1} \times \boldsymbol{\Theta}_{2}$ at $\left[\boldsymbol{\alpha}^{* \top}, \boldsymbol{\beta}^{* \top}\right]^{\top} \in \stackrel{\circ}{\Theta}_{1} \times \stackrel{\circ}{\Theta}_{2}$. If $\operatorname{det}\left[\partial^{2} s(\boldsymbol{\alpha}, \boldsymbol{\beta}) / \partial \boldsymbol{\alpha} \partial \boldsymbol{\alpha}^{\top}\right] \neq 0$ and $\operatorname{det}\left[\partial^{2} v(\boldsymbol{\alpha}, \boldsymbol{\beta}) / \partial \boldsymbol{\beta} \partial \boldsymbol{\beta}^{\top}\right] \neq 0$ for all $\left[\boldsymbol{\alpha}^{\top}, \boldsymbol{\beta}^{\top}\right]^{\top} \in \stackrel{\circ}{\boldsymbol{\Theta}}_{1} \times \stackrel{\circ}{\Theta}_{2}$, then

$$
\left[\begin{array}{l}
\widehat{\boldsymbol{\alpha}}\left(\boldsymbol{\beta}^{*}\right) \\
\widehat{\boldsymbol{\beta}}\left(\boldsymbol{\alpha}^{*}\right)
\end{array}\right] \underset{n \rightarrow \infty}{\stackrel{P}{\longrightarrow}}\left[\begin{array}{l}
\boldsymbol{\alpha}^{*} \\
\boldsymbol{\beta}^{*}
\end{array}\right]
$$

Proof. Let us define $\varphi(\boldsymbol{\alpha}, \boldsymbol{\beta}):=\partial s(\boldsymbol{\alpha}, \boldsymbol{\beta}) / \partial \boldsymbol{\alpha}$ and $\varrho(\boldsymbol{\alpha}, \boldsymbol{\beta}):=\partial v(\boldsymbol{\alpha}, \boldsymbol{\beta}) / \partial \boldsymbol{\beta}$. Since $\varphi\left(\boldsymbol{\alpha}^{*}(\boldsymbol{\beta}), \boldsymbol{\beta}\right)=\mathbf{0}$ for all $\boldsymbol{\beta} \in \stackrel{\circ}{\boldsymbol{\Theta}}_{2}$, then for all $\boldsymbol{\beta} \in \stackrel{\circ}{\Theta}_{2}$ by the implicit function theorem, there exists a unique function $\phi \in \mathcal{C}^{1}$ on the open surrounding of $\boldsymbol{\beta}$ such that $\phi(\boldsymbol{\beta})=\boldsymbol{\alpha}$. Due to this uniqueness, $\phi(\cdot) \equiv \boldsymbol{\alpha}^{*}(\cdot)$.

Similarly, for all $\boldsymbol{\alpha} \in \stackrel{\circ}{\Theta}_{1}, \varrho\left(\boldsymbol{\alpha}, \boldsymbol{\beta}^{*}(\boldsymbol{\alpha})\right)=\mathbf{0}$ and, thus, there exists a unique function $\rho \in \mathcal{C}^{1}$ on the open surrounding of $\boldsymbol{\alpha}$ such that $\rho(\boldsymbol{\alpha})=\boldsymbol{\beta}$. Hence, $\rho(\cdot) \equiv \boldsymbol{\beta}^{*}(\cdot) \in \mathcal{C}^{1}\left(\stackrel{\circ}{\Theta}_{1}\right)$.

Consequently, since $\varphi\left(\boldsymbol{\alpha}^{*}\left(\boldsymbol{\beta}^{*}\right), \boldsymbol{\beta}^{*}\right)=0$ and $\varrho\left(\boldsymbol{\alpha}^{*}, \boldsymbol{\beta}^{*}\left(\boldsymbol{\alpha}^{*}\right)\right)=0$, then $\boldsymbol{\alpha}^{*}\left(\boldsymbol{\beta}^{*}\right)=\boldsymbol{\beta}^{*}$ and $\boldsymbol{\beta}^{*}\left(\boldsymbol{\alpha}^{*}\right)=\boldsymbol{\alpha}^{*}$. Nevertheless by Theorems 1 and 2 ,

$$
\left[\begin{array}{c}
\widehat{\boldsymbol{\alpha}}\left(\boldsymbol{\beta}^{*}\right) \\
\widehat{\boldsymbol{\beta}}\left(\boldsymbol{\alpha}^{*}\right)
\end{array}\right] \underset{n \rightarrow \infty}{\stackrel{\mathrm{P}}{\longrightarrow}}\left[\begin{array}{c}
\boldsymbol{\alpha}^{*}\left(\boldsymbol{\beta}^{*}\right) \\
\boldsymbol{\beta}^{*}\left(\boldsymbol{\alpha}^{*}\right)
\end{array}\right]=\left[\begin{array}{c}
\boldsymbol{\alpha}^{*} \\
\boldsymbol{\beta}^{*}
\end{array}\right] .
$$

Variance parameter $\boldsymbol{\beta}$ can be viewed as a nuisance parameter when estimating the mean parameter $\boldsymbol{\alpha}$ and vice-versa. The idea of joint estimation of $\left[\boldsymbol{\alpha}^{\top}, \boldsymbol{\beta}^{\top}\right]^{\top}$ is to alternately perform partial optimizations from Definition 2 , In fact, we iteratively estimate $\boldsymbol{\alpha}$ given the fixed value of $\boldsymbol{\beta}$ and, consequently, we estimate $\boldsymbol{\beta}$ given the fixed value of $\boldsymbol{\alpha}$ (obtained from previous step). This two steps are repeated in turns until almost no change in consecutive estimates of $\left[\boldsymbol{\alpha}^{\top}, \boldsymbol{\beta}^{\top}\right]^{\top}$, see Algorithm 1. Based on Corollary 3, it is believed that each turn will bring our iterated estimates closer to the true unknown parameter values. Moreover, Algorithm 1 can be modified: the initial value of $\boldsymbol{\alpha}^{(0)}$ could be required on the input instead of $\boldsymbol{\beta}^{(0)}$ and the whole iteration procedure would start with the estimation of $\boldsymbol{\beta}^{(1)}$ instead of $\boldsymbol{\alpha}^{(1)}$.

In order to demonstrate the finite-sample performance of the CLS estimates, a small simulation study is provided. 200 triangles with $n=11$ accident years are simulated. The claim amounts in the first column of each 
Algorithm 1 Iterative conditional least squares estimation of $\boldsymbol{\alpha}$ and $\boldsymbol{\beta}$.

Input: Cumulative claims triangle $\left\{Y_{i, j}\right\}_{i, j=1}^{n, n+1-i}$, mean and variance functions $\mu$ and $\sigma$, initial (starting) parameter value $\boldsymbol{\beta}^{(0)}$, maximum number of iterations $M$, and convergence precision $\epsilon$.

Output: CLS parameter estimates $\widehat{\boldsymbol{\alpha}}$ and $\widehat{\boldsymbol{\beta}}$, fitted residuals $\left\{\widehat{\varepsilon}_{i, j}\right\}_{i=1, j=2}^{n-1, n+1-i}$.

1: $m \leftarrow 1$ and $\boldsymbol{\alpha}^{(0)} \leftarrow \mathbf{0}$

2: $\boldsymbol{\alpha}^{(1)} \leftarrow \arg \min _{\boldsymbol{\alpha} \in \boldsymbol{\Theta}_{1}} M_{n}\left(\boldsymbol{\alpha}, \boldsymbol{\beta}^{(0)}\right)$

3: $\boldsymbol{\beta}^{(1)} \leftarrow \arg \min _{\boldsymbol{\beta} \in \boldsymbol{\Theta}_{2}} V_{n}\left(\boldsymbol{\alpha}^{(1)}, \boldsymbol{\beta}\right)$

4: while $m \leq M$ and $\left\|\left[\boldsymbol{\alpha}^{(m) \top}, \boldsymbol{\beta}^{(m) \top}\right]^{\top}-\left[\boldsymbol{\alpha}^{(m-1) \top}, \boldsymbol{\beta}^{(m-1) \top}\right]^{\top}\right\|>\epsilon$ do

5: $\quad \boldsymbol{\alpha}^{(m+1)} \leftarrow \arg \min _{\boldsymbol{\alpha} \in \boldsymbol{\Theta}_{1}} M_{n}\left(\boldsymbol{\alpha}, \boldsymbol{\beta}^{(m)}\right)$

6: $\quad \boldsymbol{\beta}^{(m+1)} \leftarrow \arg \min _{\boldsymbol{\beta} \in \boldsymbol{\Theta}_{2}} V_{n}\left(\boldsymbol{\alpha}^{(m+1)}, \boldsymbol{\beta}\right)$

7: $\quad m \leftarrow m+1$

8: end while

9: $\widehat{\boldsymbol{\alpha}} \leftarrow \boldsymbol{\alpha}^{(m)}$ and $\widehat{\boldsymbol{\beta}} \leftarrow \boldsymbol{\beta}^{(m)}$

10: for $i=1$ to $n-1$ do

11: $\quad$ for $j=2$ to $n+1-i$ do

12: $\quad \widehat{\varepsilon}_{i, j} \leftarrow\left[Y_{i, j}-\mu\left(Y_{i, j-1}, \widehat{\boldsymbol{\alpha}}, j\right)\right] / \sigma\left(Y_{i, j-1} \widehat{\boldsymbol{\beta}}, j\right)$

13: $\quad$ end for

14: end for

triangle are iid gamma distributed with mean and also variance equal to $10^{5}$. The mean and variance functions of the CMV model are chosen as in the forthcoming real data example (Section 7), see CMV structure (11). The marginal distribution of the errors is the standard gaussian and the bivariate copula is the Gumbel copula with parameter 2. The true unknown parameter values together with the sample means and sample standard deviations of their CLS estimates based on 200 triangle samples are shown in Table 2. All four sample means are close to the corresponding true values, which is in concordance with the asymptotic theory derived above. 


\begin{tabular}{lrrrr}
\hline Parameter & $\alpha_{1}$ & $\alpha_{2}$ & $\beta_{1}$ & $\beta_{2}$ \\
\hline True value & 2.000 & 1.000 & 100.000 & 0.500 \\
Mean & 2.001 & 1.001 & 101.214 & 0.508 \\
Standard deviation & 0.029 & 0.031 & 46.405 & 0.131 \\
\hline
\end{tabular}

Table 2: Sample means and sample standard deviations of 200 CLS estimates for the true parameter values (based on simulations).

\subsection{Estimation of Dependence Structure}

The second stage of the parameter estimation process involves the estimation of the whole dependence structure in the claims triangle. Indeed, the strict stationarity of the first order Markov process imposed on the CMV model errors (Assumption C) together with Sklar's theorem arrange that only the copula parameter and the marginal distribution of the errors are necessary to know the bivariate distribution of two in row neighbouring errors.

Since the estimates of the CMV model parameters are already available, one can estimate the unknown marginal distribution function $G_{\boldsymbol{\alpha}, \boldsymbol{\beta}}$ of CMV model errors $\varepsilon_{i, j}(\boldsymbol{\alpha}, \boldsymbol{\beta})$ non-parametrically by the empirical distribution function

$$
\widehat{G}_{n}(e)=\frac{1}{n(n-1) / 2+1} \sum_{i=1}^{n-1} \sum_{j=2}^{n+1-i} \mathcal{I}\left\{\widehat{\varepsilon}_{i, j}(\widehat{\boldsymbol{\alpha}}, \widehat{\boldsymbol{\beta}}) \leq e\right\},
$$

of the fitted residuals

$$
\widehat{\varepsilon_{i, j}}(\widehat{\boldsymbol{\alpha}}, \widehat{\boldsymbol{\beta}})=\frac{Y_{i, j}-\mu\left(Y_{i, j-1}, \widehat{\boldsymbol{\alpha}}, j\right)}{\sigma\left(Y_{i, j-1}, \widehat{\boldsymbol{\beta}}, j\right)} .
$$

The consistency of the CMV model parameter estimates $\widehat{\boldsymbol{\alpha}}$ and $\widehat{\boldsymbol{\beta}}$ ensures that the fitted residuals $\widehat{\varepsilon}_{i, j}(\widehat{\boldsymbol{\alpha}}, \widehat{\boldsymbol{\beta}})$ are reasonable predictors of the unknown nonobservable errors $\varepsilon_{i, j}(\boldsymbol{\alpha}, \boldsymbol{\beta})$. Algorithm 1 also provides the fitted residuals as a side product.

Assumption $\mathrm{C}$ demands a prior knowledge of parametric copula up to its unknown parameter $\gamma$. Nevertheless in practical applications, one needs to perform a copula goodness-of-fit in order to choose a suitable copula. Assuming that we know the bivariate copula function $C(\cdot, \cdot ; \gamma)$, copula parameter 
$\gamma$ is estimated by the quasi-likelihood method.

Having in mind that rows of errors $\left[\varepsilon_{i, 1}(\boldsymbol{\alpha}, \boldsymbol{\beta}), \ldots, \varepsilon_{i, n+1-i}(\boldsymbol{\alpha}, \boldsymbol{\beta})\right]$ are independent for all $i$, the full log-likelihood for copula parameter $\gamma$ with respect to $(3)$ is

$$
\begin{aligned}
\mathcal{L}(\boldsymbol{\gamma})=\sum_{i=1}^{n-2} \sum_{j=2}^{n+1-i} & \log g_{\boldsymbol{\alpha}, \boldsymbol{\beta}}\left(\varepsilon_{i, j}(\boldsymbol{\alpha}, \boldsymbol{\beta})\right) \\
& \quad+\sum_{i=1}^{n-2} \sum_{j=3}^{n+1-i} \log c\left(G_{\boldsymbol{\alpha}, \boldsymbol{\beta}}\left(\varepsilon_{i, j-1}(\boldsymbol{\alpha}, \boldsymbol{\beta})\right), G_{\boldsymbol{\alpha}, \boldsymbol{\beta}}\left(\varepsilon_{i, j}(\boldsymbol{\alpha}, \boldsymbol{\beta})\right) ; \boldsymbol{\gamma}\right)
\end{aligned}
$$

Ignoring the first term in $\mathcal{L}(\gamma)$ and replacing $\varepsilon^{\prime}$ s and $G_{\boldsymbol{\alpha}, \boldsymbol{\beta}}$ by their estimated counterparts $\widehat{\varepsilon}$ 's and $\widehat{G}_{n}$, parameter $\gamma$ can be estimated by the socalled canonical maximum likelihood, i.e., maximizing the partial (pseudo) log-likelihood:

$$
\begin{aligned}
\widehat{\boldsymbol{\gamma}} & =\arg \max _{\boldsymbol{\gamma}} \widetilde{\mathcal{L}}(\boldsymbol{\gamma}) \\
\widetilde{\mathcal{L}}(\boldsymbol{\gamma}) & =\sum_{i=1}^{n-2} \sum_{j=3}^{n+1-i} \log c\left(\widehat{G}_{n}\left(\widehat{\varepsilon}_{i, j-1}(\widehat{\boldsymbol{\alpha}}, \widehat{\boldsymbol{\beta}})\right), \widehat{G}_{n}\left(\widehat{\varepsilon}_{i, j}(\widehat{\boldsymbol{\alpha}}, \widehat{\boldsymbol{\beta}})\right) ; \boldsymbol{\gamma}\right)
\end{aligned}
$$

The correctness of this approach was shown by Chen and Fan $(2006)$, because the consistency of the canonical likelihood estimate of copula parameter $\gamma$ was proved under Assumption C. Here, the unknown unobservable CMV model errors are just replaced by the fitted residuals based on the consistent CMV model parameter estimates. Algorithm 2 encapsulates the way of getting copula parameter estimate.

Algorithm 2 Copula parameter $\gamma$ estimation by pseudo-likelihood.

Input: Fitted residuals $\left\{\widehat{\varepsilon}_{i, j}(\widehat{\boldsymbol{\alpha}}, \widehat{\boldsymbol{\beta}})\right\}_{i=1, j=2}^{n-1, n+1-i}$ and copula density $c(\cdot, \cdot ; \boldsymbol{\gamma})$.

Output: Copula parameter estimate $\widehat{\gamma}$.

1: marginal ecdf $\widehat{G}_{n}(e) \leftarrow \frac{1}{n(n-1) / 2+1} \sum_{i=1}^{n-1} \sum_{j=2}^{n+1-i} \mathcal{I}\left\{\widehat{\varepsilon}_{i, j}(\widehat{\boldsymbol{\alpha}}, \widehat{\boldsymbol{\beta}}) \leq e\right\}$

$2: \widehat{\boldsymbol{\gamma}} \leftarrow \arg \max _{\boldsymbol{\gamma}} \sum_{i=1}^{n-2} \sum_{j=3}^{n+1-i} \log c\left(\widehat{G}_{n}\left(\widehat{\varepsilon}_{i, j-1}(\widehat{\boldsymbol{\alpha}}, \widehat{\boldsymbol{\beta}})\right), \widehat{G}_{n}\left(\widehat{\varepsilon}_{i, j}(\widehat{\boldsymbol{\alpha}}, \widehat{\boldsymbol{\beta}})\right) ; \boldsymbol{\gamma}\right)$

Chen and Fan (2006) also remarked that the empirical distribution function $\widehat{G}_{n}$ can be smoothed by kernels as an alternative estimate of the marginal 
distribution of errors. This can especially be helpful in case of a smaller number of residuals available.

Now, the whole estimation procedure became a semiparametric one.

\section{Prediction of Reserves and Estimating Their Distribution}

The main goals in actuarial reserving are prediction of reserves $R_{i}^{(n)}$ and, consequently, estimation of the reserves' distribution, e.g., in order to obtain quantiles - $99.5 \%$ quantile for the Solvency II purposes (VaR calculation).

A predictor for reserve $R_{i}^{(n)}$ can be defined as

$$
\widehat{R}_{i}^{(n)}=\widehat{Y}_{i, n}-Y_{i, n+1-i} \text {. }
$$

Therefore, finding a predictor $\widehat{Y}_{i, n}$ for $Y_{i, n}$ is crucial. One naive proposal is to predict $Y_{i, j}$ as conditional mean of previous claim amount $Y_{i, j-1}$ with the plugged-in CMV model parameter estimates

$$
\check{Y}_{i, j}:=\left.\mathrm{E}_{\boldsymbol{\alpha}, \boldsymbol{\beta}}\left[Y_{i, j} \mid Y_{i, n+1-i}\right]\right|_{\boldsymbol{\alpha}=\widehat{\boldsymbol{\alpha}}, \boldsymbol{\beta}=\widehat{\boldsymbol{\beta}}}, \quad i=2, \ldots, n, j=n+2-i, \ldots, n .
$$

This approach gives $\check{Y}_{i, j}=\mu\left(\check{Y}_{i, j-1}, \widehat{\boldsymbol{\alpha}}, j\right), i+j>n+1$. In spite of that, such approach would be eligible only if prediction (9) was unbiased or at least asymptotically unbiased, which is not justified.

\subsection{Semiparametric Bootstrap}

Prediction of unobserved claims may be done in a telescopic way based on the CMV model formulation: start with the diagonal element $Y_{i, n+1-i}$ and predict $Y_{i, j}, j>n+1-i$ stepwise in each row

$$
\begin{aligned}
& \widehat{Y}_{i, j}=Y_{i, j}, \quad i+j \leq n+1 \\
& \widehat{Y}_{i, j}=\mu\left(\widehat{Y}_{i, j-1}, \widehat{\boldsymbol{\alpha}}, j\right)+\sigma\left(\widehat{Y}_{i, j-1}, \widehat{\boldsymbol{\beta}}, j\right) \widetilde{\varepsilon}_{j}, \quad i+j>n+1 .
\end{aligned}
$$

Errors $\widetilde{\varepsilon}_{j}$ are simulated from the fitted residuals. One convenient resampling procedure is the semiparametric bootstrap which takes advantage of the fact that $\varepsilon_{i, j}(\boldsymbol{\alpha}, \boldsymbol{\beta})=G_{\boldsymbol{\alpha}, \boldsymbol{\beta}}^{-1}\left(X_{j}\right)$ for all $i$ (due to the independent rows), where $\left\{X_{j}\right\}_{j=2}^{n}$ is a stationary first-order Markov process with the copula $C\left(x_{1}, x_{2} ; \gamma\right)$ being the joint distribution of $\left[X_{j-1}, X_{j}\right]$.

When errors $\widetilde{\varepsilon}_{j}$ are simulated sufficiently many times, the empirical (bootstrap) distribution of $\widehat{Y}_{i, n}$ is obtained, which should mimic the true unknown 
distribution of $Y_{i, n}$. Thereafter, an estimate of reserves' distribution is acquired and some imported quantities of the reserves can be easily calculated, e.g., mean, variance, or quantiles of the reserves. The whole procedure is summed up in Algorithm 3 .

\section{Real Data Analysis}

The proposed method is illustrated on a data set from Zehnwirth and Barnett (2000). Here, data for 11 accident years are available $(n=11)$. The data analyses are conducted in $\mathrm{R}$ program ( $\mathrm{R}$ Core Team, 2012).

Several candidates for the mean and variance function $\mu$ and $\sigma$ were considered (see Section 3). The CLS were used to estimate the parameters of the CMV model. By inspecting the fitted residuals graphically and comparing the residuals' mean square error, the following structure of the CMV model was taken into account

$$
\begin{aligned}
& \mu\left(Y_{i, j-1}, \boldsymbol{\alpha}, j\right)=\left(1+\alpha_{1} \alpha_{2} j^{-1-\alpha_{2}} \exp \left\{\alpha_{1} j^{-\alpha_{2}}\right\}\right) Y_{i, j-1}, \\
& \sigma\left(Y_{i, j-1}, \boldsymbol{\beta}, j\right)=\beta_{1} \exp \left\{-\beta_{2} j\right\} \sqrt{Y_{i, j-1}} .
\end{aligned}
$$

The CLS estimates are $\widehat{\alpha}_{1}=2.033, \widehat{\alpha}_{2}=1.106, \widehat{\beta}_{1}=109.8$, and $\widehat{\beta}_{2}=0.4053$. Figure 1 shows the fitted residuals for the CMV model structure (11).

There is still some slight pattern (trend) not captured by mean and variance parametric part of the model (Figure 1), but that will be modeled by the residuals' dependence. Kendall $\tau$ for the pairs of consecutive residuals $\left\{\left[\widehat{\varepsilon}_{i, j-1}(\widehat{\boldsymbol{\alpha}}, \widehat{\boldsymbol{\beta}}), \widehat{\varepsilon}_{i, j}(\widehat{\boldsymbol{\alpha}}, \widehat{\boldsymbol{\beta}})\right]\right\}_{i=1, j=3}^{n-2, n+1-i}$ equals 0.43 , which indicates at least mild dependence.

Three Archimedean copulae (Clayton, Frank, and Gumbel) together with Gaussian and Student $t_{5}$-copula (the degrees of freedom were set in order to have finite fourth moment) were considered for modeling the dependence among the errors. We have performed the $S_{n}^{(C)}$ goodness-of-fit test proposed by Genest et al. (2009, Section 4). This test is preferred in our case for two reasons: it was regarded by the above cited power study as a recommendable test and it relies on Rosenblatt's transform (a single bootstrap is enough to approximate the null distribution of the test statistic and extract $p$-values). The Gumbel copula $(\widehat{\gamma}=1.776)$ was chosen according to the goodness-of-fit test (providing the highest $p$-value equal 0.30). The Gumbel copula exhibits strong right tail dependence and relatively weak left tail dependence. In our data set, the transformed residuals - transformed by the residuals' marginal 
Algorithm 3 Predictions of claims reserves and estimation of their distributions by semiparametric bootstrap.

Input: Latest diagonal of cumulative claims $\left\{Y_{i, n+1-i}\right\}_{i=2}^{n}$, empirical distribution function $\widehat{G}_{n}$ of residuals, parametric mean and variance functions $\mu$ and $\sigma$, inverse of conditional parametric copula $C_{2 \mid 1}^{-1}$, parameter estimates $\widehat{\boldsymbol{\alpha}}, \widehat{\boldsymbol{\beta}}$, and $\widehat{\boldsymbol{\gamma}}$, number of bootstrap simulations $B$.

Output: Empirical bootstrap distribution of predicted reserves $\widehat{R}_{i}^{(n)}$, i.e., the empirical distribution where probability mass $1 / B$ concentrates at each of ${ }_{(1)} \widehat{R}_{i}^{(n)}, \ldots,{ }_{(B)} \widehat{R}_{i}^{(n)}, i=2, \ldots, n$.

1: for $b=1$ to $B$ do

2: $\quad$ generate $n-1$ independent $U(0,1)$ random variables $\left\{X_{j}\right\}_{j=2}^{n}$

3: $\quad{ }_{(b)} U_{2} \leftarrow X_{2}$

4: $\left.\quad{ }_{(b)} \widehat{\varepsilon}_{2} \leftarrow \widehat{G}_{n}^{-}{ }_{\left({ }_{(b)}\right.} U_{2}\right) / / \widehat{G}_{n}^{-}$is the empirical quantile function

5: $\quad$ for $j=3$ to $n$ do

6: $\quad{ }_{(b)} U_{j} \leftarrow C_{2 \mid 1}^{-1}\left(\left.X_{j}\right|_{(b)} U_{j-1} ; \widehat{\gamma}\right)$

7: $\quad\left({ }_{(b)} \widehat{\varepsilon}_{j} \leftarrow \widehat{G}_{n}^{-}\left({ }_{(b)} U_{j}\right)\right.$

8: $\quad$ end for

9: end for

10: center bootstrap residuals ${ }_{(b)} \widetilde{\varepsilon}_{j} \leftarrow{ }_{(b)} \widehat{\varepsilon}_{j}-\frac{1}{n-1} \sum_{l=2}^{n}(b) \widehat{\varepsilon}_{l}, b=1, \ldots, B$

11: for $i=2$ to $n$ do

12: $\quad$ for $b=1$ to $B$ do $/ /$ repeat to obtain empirical distribution of $\widehat{R}_{i}^{(n)}$

13:

(b) $\widehat{Y}_{i, n+1-j} \leftarrow Y_{i, n+1-i}$

14: $\quad$ for $j=n+2-i$ to $n$ do

15:

16 :

$$
\left.{ }_{(b)} \widehat{Y}_{i, j} \leftarrow \mu\left(_{(b)} \widehat{Y}_{i, j-1}, \widehat{\boldsymbol{\alpha}}, j\right)+\sigma{ }_{(b)} \widehat{Y}_{i, j-1}, \widehat{\boldsymbol{\beta}}, j\right)_{(b)} \widetilde{\varepsilon}_{j}
$$

end for

17 :

$$
{ }_{(b)} \widehat{R}_{i}^{(n)} \leftarrow{ }_{(b)} \widehat{Y}_{i, n}-Y_{i, n+1-i}
$$

\section{end for}

19: end for

empirical distribution function $\widehat{G}_{n}$ (having uniform margins)- seem to be strongly correlated at high values but less correlated at low values (see Fig- 


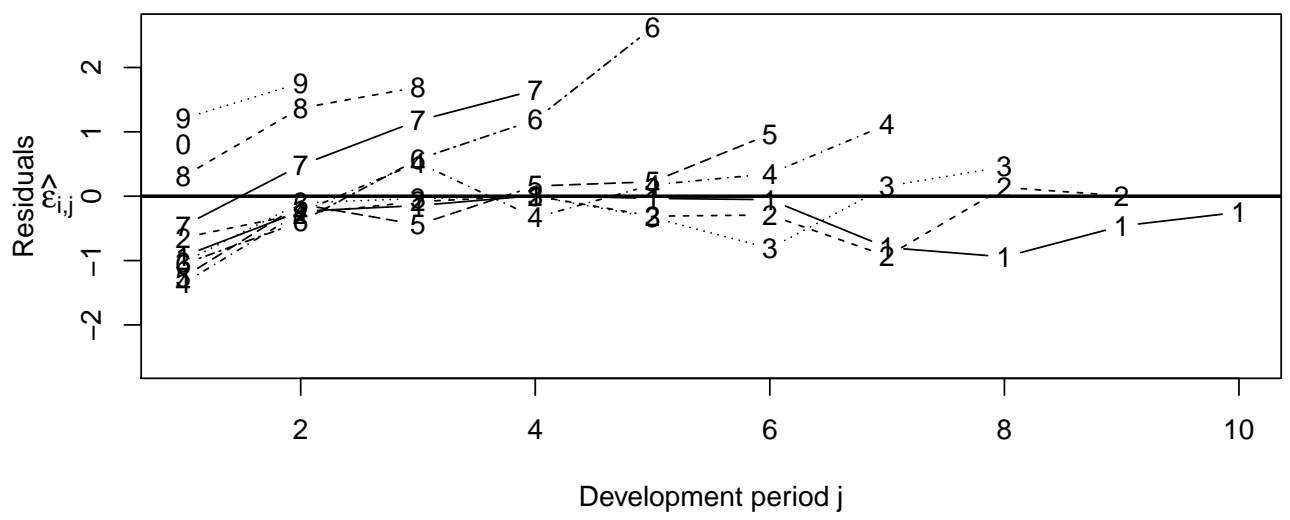

Figure 1: Residuals of the CMV model (11) with a common accident year are connected by lines ( 0 stands for the accident year $i=10)$.

ure 21). Then, the Gumbel copula is indeed an appropriate choice. The pairs of original residuals $\left[\widehat{\varepsilon}_{i, j-1}(\widehat{\boldsymbol{\alpha}}, \widehat{\boldsymbol{\beta}}), \widehat{\varepsilon}_{i, j}(\widehat{\boldsymbol{\alpha}}, \widehat{\boldsymbol{\beta}})\right]$ are shown in Figure 2 as well. Moreover, Figure 2 contains the density for the Gumbel copula $C(\cdot, \cdot ; \widehat{\gamma})$ and the first 500 pairs of the bootstrapped residuals, which should jointly "behave" like the original ones.

As a benchmark to the CMV model with copula Assumption C, the classical bootstrap version of chain ladder (England and Verrall, 2002, A3.1) is chosen. It has some disadvantages, which can be overcome by our approach: the number of parameters (development factors) depending on the sample size, some parameters (the last development factor) estimated by just ratio of two numbers (yielding zero sample variance), questionable consistency of the estimates (conditional consistency recently proved by Pešta and Hudecová (2012)), and the non-realistic assumption of independence of the residuals.

The results of the proposed approach-conditional least squares with copula (CLSC) - and the traditional one - bootstrapped chain ladder (BCL) - are compared numerically in Table 3 and graphically in Figure 3 . In both cases, the number of bootstrap replications is $B=5000$.

For each particular accident year and in total as well, the CLSC approach provides slightly smaller predictions of reserves than the BCL one. But even more important is that the estimates of the reserves' distribution are less 

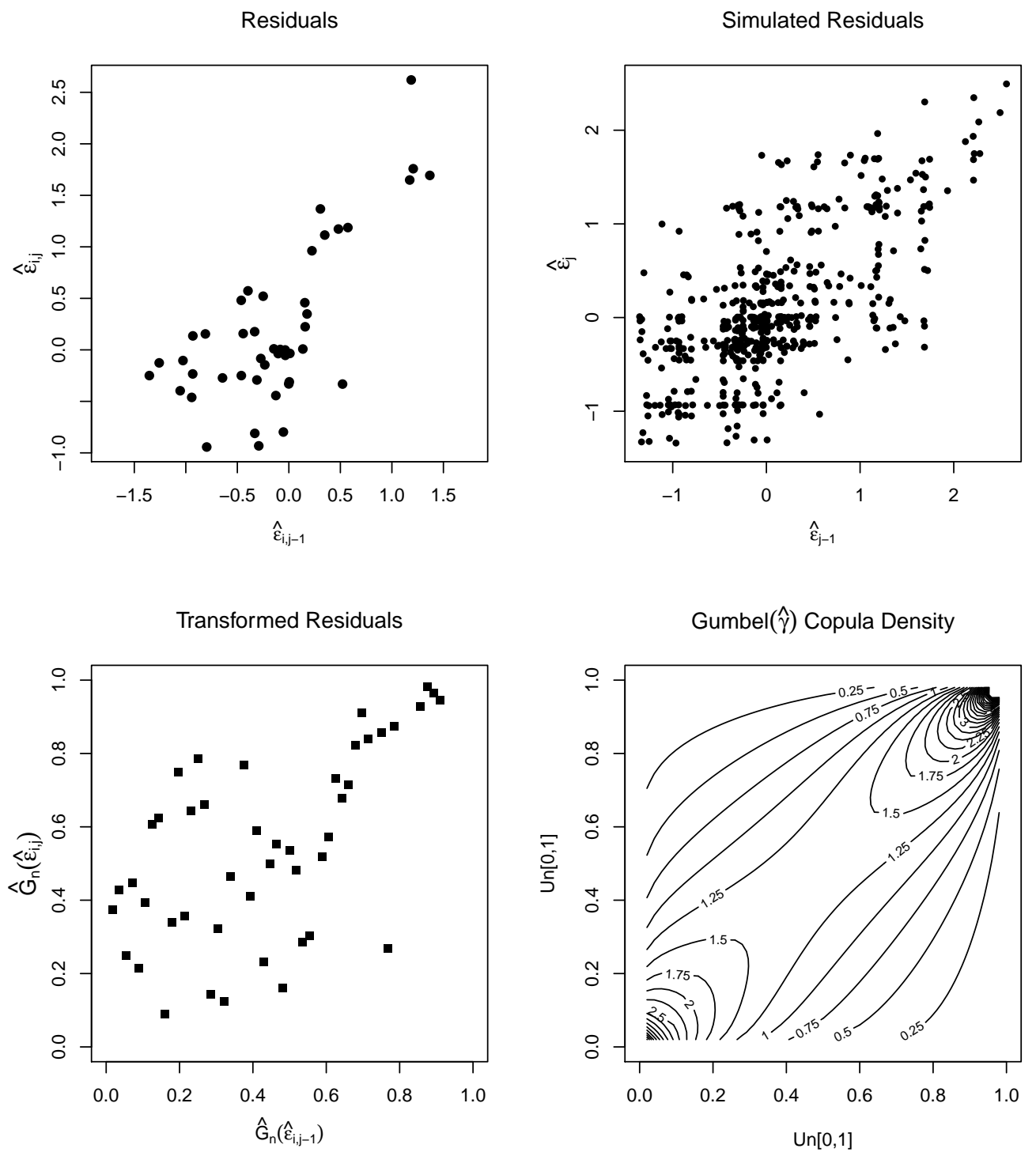

Figure 2: Pairs of original fitted residuals $\left[\widehat{\varepsilon}_{i, j-1}(\widehat{\boldsymbol{\alpha}}, \widehat{\boldsymbol{\beta}}), \widehat{\varepsilon}_{i, j}(\widehat{\boldsymbol{\alpha}}, \widehat{\boldsymbol{\beta}})\right]$ (top left), transformed residuals by the marginal empirical distribution function $\widehat{G}_{n}$ (bottom left), estimated density $c(\cdot, \cdot ; \widehat{\gamma})$ of the Gumbel copula (bottom right), simulated (bootstrapped) residuals $\left[(b) \widehat{\varepsilon}_{j-1},(b) \widehat{\varepsilon}_{j}\right]$ from the Gumbel copula (top right).

volatile (e.g., smaller standard error or smaller difference between the third and the first quartile) and have lighter right tails (and, hence, smaller high 


\begin{tabular}{|c|c|c|c|c|c|c|c|c|}
\hline \multirow[t]{2}{*}{$i$} & \multicolumn{2}{|c|}{ Reserve } & \multicolumn{2}{|c|}{ Standard error } & \multicolumn{2}{|c|}{ 95\%-quantile } & \multicolumn{2}{|c|}{ 99.5\%-quantile } \\
\hline & BCL & CLSC & BCL & CLSC & BCL & CLSC & BCL & CLSC \\
\hline 2 & 14 & 15 & 5 & 1 & 24 & 16 & 31 & 17 \\
\hline 3 & 38 & 8 & 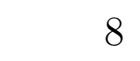 & 2 & 51 & 42 & 61 & 44 \\
\hline 4 & 64 & 6 & 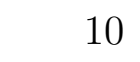 & 3 & 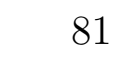 & 70 & 91 & 73 \\
\hline 5 & 100 & 10 & 12 & - & 1 & 109 & 132 & 114 \\
\hline 6 & 144 & 145 & 14 & 6 & 168 & 156 & 183 & 162 \\
\hline 7 & 211 & 208 & 17 & 8 & 241 & 222 & 261 & 231 \\
\hline 8 & 385 & 373 & 24 & 13 & 427 & 396 & 450 & 411 \\
\hline 9 & 765 & $7:$ & 00 & 24 & 831 & 769 & 868 & 794 \\
\hline 10 & 1362 & 1294 & 59 & 44 & 1464 & 1370 & 1531 & 1413 \\
\hline 11 & 2195 & 2153 & 111 & 81 & 2389 & 2293 & 2508 & 2374 \\
\hline Total & 5279 & 5122 & 172 & 115 & 5565 & 5317 & 5751 & 5432 \\
\hline
\end{tabular}

Table 3: BCL and CLSC reserving results for Zehnwirth and Barnett (2000) data (values in thousands).

quantiles) for the CLSC compared to the BCL. By virtue of such unambiguous outcomes, wasting number of parameters and impetuous neglecting of present dependence in data really matters.

Additionally, an interesting finding comes out, when the CMV model with copula approach is compared to the approach suggested by Hudecová and Pešta (2013), where the dependence is modeled by generalized estimating equations (GEE). Such a different method provides very similar results. For instance, the GEE (with logarithmic link function, AR(1) correlation structure, and linear variance function) provides coefficient of variation $(\mathrm{CoV})$ 1.95 for the Zehnwirth and Barnett (2000) data, whereas the CoV in case of the CLSC method is equal to 1.99. For a complete comparison, the $\mathrm{CoV}$ for the traditional nonparametric BCL, which disregards dependence, equals 3.29 . 



Figure 3: Reserves distribution based on BCL (light gray) and CLSC (dark gray). 


\section{Conclusions and Discussion}

This paper proposes the conditional mean and variance $(C M V)$ time series model with innovations being a stationary first-order Markov process. Such a framework is demonstrated to be suitable for stochastic claims reserving in general insurance. It brings several advantages, which should be though of a natural relaxation of some too restrictive assumptions in the traditional methods. Indeed in contrast to the classical reserving techniques, a large number of the mean and variance structures for claims is allowed by the general model's definition yielding a very flexible modeling approach, relatively smaller number of model parameters not depending on the number of development periods is required, and time series innovations (errors) are not considered as independent. All of the previously mentioned benefits contribute to the increase in precision of the claims reserves' prediction (e.g., smaller variability, lighter tails). Furthermore, claims forecasting beyond the last observed development period becomes straightforward.

The conditional least squares (CLS) together with the copula approach provide parameter estimates of the assumed model. Moreover, semiparametric bootstrap, as an extension based on the CLS and copulae modeling purview, is used in order to estimate the whole distribution of predicted reserves.

Generally, the consistency of every bootstrap procedure relies on the consistency of the parameter estimates. In the proposed approach, the consistency of the CLS parameter estimates in the CMV model is shown, which gives validity for the bootstrapping. This brings the CMV model to the fore, because the consistency for the chain ladder development factors (together with the necessary and sufficient condition), that has recently been proved by Pešta and Hudecová (2012), is only conditional, which makes the further usage more complicated.

Our approach can also be robustified in the sense that not only the last observed diagonal of claims is taken into account for the reserves' prediction as in (10), but the telescopic prediction begins from each of the observed claims. Finally, the procedure of predicting reserves can even be generalized by incorporating time-varying copulae, which enables to omit the stationary dependence structure, although, it requires more complicated setup. 


\section{Acknowledgments}

The research of Michal Pešta was supported by the Czech Science Foundation project GAČR No. P201/13/12994P. Financial support through CRC 649 "Ökonomisches Risiko" is gratefully acknowledged by Ostap Okhrin.

\section{References}

Antonio, K., Beirlant, J., 2007. Actuarial statistics with generalized linear mixed models. Insurance: Mathematics and Economics 40, 58-76.

Bierens, H. J., 1994. Topics in Advanced Econometrics: Estimation, Testing and Specification of Cross-section and Time Series Models. Cambridge University Press, New York, NY.

Bollerslev, T., 2010. Glossary to ARCH (GARCH). In: Bollerslev, T., Russell, J. R., Watson, M. W. (Eds.), Volatility and Time Series Econometrics: Essays in Honor of Robert F. Engle. Oxford University Press, Oxford.

Buchwalder, M., Bühlmann, H., Merz, M., Wüthrich, M. V., 2006. The mean square error of prediction in the chain ladder reserving method (Mack and Murphy revisited). ASTIN Bulletin 36 (2), 521-542.

Chen, X., Fan, Y., 2006. Estimation of copula-based semiparametric time series models. Journal of Econometrics 130 (2), 307-335.

Davidson, J., 1994. Stochastic Limit Theory: An Introduction for Econometricians. Oxford University Press, New York, NY.

England, P. D., Verrall, R. J., 2002. Stochastic claims reserving in general insurance. British Actuarial Journal 8, 443-544.

Genest, C., Rémillard, B., Beaudoin, D., 2009. Goodness-of-fit tests for copulas: A review and a power study. Insurance: Mathematics and Economics 44 (2), 199-213.

Hudecová, Š., Pešta, M., 2013. Modeling dependencies in claims reserving with GEE. Submitted http://arxiv.org/abs/1306.3768.

Mack, T., 1993. Distribution-free calculation of the standard error of chain ladder reserve estimates. ASTIN Bulletin 23 (2), 213-225. 
Patton, A. J., 2012. A review of copula models for economic time series. Journal of Multivariate Analysis 110, 4-18.

Pešta, M., Hudecová, Š., 2012. Asymptotic consistency and inconsistency of the chain ladder. Insurance: Mathematics and Economics 51 (2), 472-479.

R Core Team, 2012. R: A Language and Environment for Statistical Computing. R Foundation for Statistical Computing, Vienna, Austria.

URL http://www.R-project.org

Sherman, R. E., 1984. Extrapolating, smoothing, and interpolating development factors. Proceedings of the Casualty Actuarial Society 72, 122-155.

Shi, P., Frees, E. W., 2011. Dependent loss reserving using copulas. ASTIN Bulletin 41 (2), 449-486.

Wüthrich, M. V., Merz, M., 2008. Stochastic claims reserving methods in insurance. Wiley finance series. John Wiley \& Sons.

Zehnwirth, B., Barnett, G., 2000. Best estimates for reserves. In: Proceedings of the Casualty Actuarial Society. No. 167. pp. 245-321. 